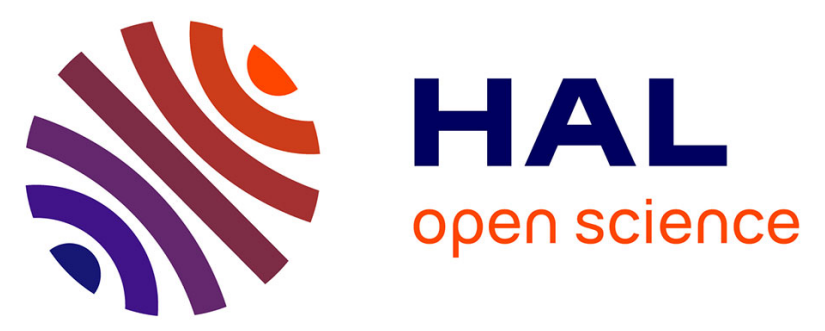

\title{
Short- and Long-Range Solvation Effects on the Transient UV-Vis Absorption Spectra of a Ru(II)-Polypyridine Complex Disentangled by Nonequilibrium Molecular Dynamics
}

Giacomo Prampolini, Francesca Ingrosso, Javier Cerezo, Alessandro Iagatti, Paolo Foggi, Mariachiara Pastore

\section{To cite this version:}

Giacomo Prampolini, Francesca Ingrosso, Javier Cerezo, Alessandro Iagatti, Paolo Foggi, et al.. Short- and Long-Range Solvation Effects on the Transient UV-Vis Absorption Spectra of a $\mathrm{Ru}(\mathrm{II})-$ Polypyridine Complex Disentangled by Nonequilibrium Molecular Dynamics. Journal of Physical Chemistry Letters, 2019, 10 (11), pp.2885-2891. 10.1021/acs.jpclett.9b00944 . hal-03445148

\author{
HAL Id: hal-03445148 \\ https://hal.science/hal-03445148
}

Submitted on 23 Nov 2021

HAL is a multi-disciplinary open access archive for the deposit and dissemination of scientific research documents, whether they are published or not. The documents may come from teaching and research institutions in France or abroad, or from public or private research centers.
L'archive ouverte pluridisciplinaire HAL, est destinée au dépôt et à la diffusion de documents scientifiques de niveau recherche, publiés ou non, émanant des établissements d'enseignement et de recherche français ou étrangers, des laboratoires publics ou privés. 


\title{
Short- and Long-Range Solvation Effects on the Transient UV-Vis Absorption Spectra of a $\mathrm{Ru}(\mathrm{II})$-polypyridine Complex Disentangled by Non-Equilibrium Molecular Dynamics
}

\author{
Giacomo Prampolini, ${ }^{a, *}$ Francesca Ingrosso, ${ }^{b, *}{ }^{2}$ avier Cerezo ${ }^{c}$ \\ Alessandro Iagatti ${ }^{d, e}$ Paolo Foggi, ${ }^{d, e, f}$ and Mariachiara Pastore ${ }^{b, *}$
}

${ }^{a}$ Istituto di Chimica dei Composti OrganoMetallici (ICCOM-CNR), Area della Ricerca, via G. Moruzzi 1, I-56124 Pisa, Italy

${ }^{b}$ Université de Lorraine \& CNRS, Laboratoire de Physique et Chimie Théoriques (LPCT),

F-54000, Nancy, France

${ }^{c}$ Departamento de Química, Facultad de Ciencias,

Universidad Autonoma de Madrid, 28049 Madrid, Spain

${ }^{d}$ European Laboratory for Non Linear Spectroscopy (LENS), Università di Firenze,

Via Nello Carrara 1, I-50019 Sesto Fiorentino, Florence, Italy

${ }^{e}$ INO-CNR, Istituto Nazionale di Ottica, Consiglio Nazionale delle Ricerche, Largo Fermi 6, I-50125 Florence, Italy

${ }^{f}$ Dipartimento di Chimica, Biologia e Biotecnologie, Università di Perugia,

Via Elce di Sotto 8, I-06123 Perugia, Italy

May 10, 2019 


\begin{abstract}
Evidences of subtle effects in the dynamic reorganization of a protic solvent in its firstand farther-neighbor shells, in response to the sudden change in the solute's electronic distribution upon excitation, are unveiled by a multi-level computational approach. By combining non-equilibrium molecular dynamics and quantum mechanical calculations, the experimental time evolution of the transient $\mathrm{T}_{1}$ absorption spectra of an heteroleptic $\mathrm{Ru}(\mathrm{II})$ polypyridine complex in ethanol or di-methyl sulfoxide solution is reproduced and rationalized in terms of both fast and slow solvent re-equilibration processes, which are found responsible for the red shift and broadening experimentally observed only in the protic medium. Solvent orientational correlation functions and a time-dependent analysis of the solvation structure confirm that the initial, fast observed red shift can be traced back to the destruction/formation of hydrogen bond networks in the first-neighbor shell, whereas the subsequent shift, evident in the [20-500] ps range and accompanied by a large broadening of the signal, is connected to a collective re-orientation of the second and farther solvation shells, which significantly changes the electrostatic embedding felt by the excited solute.
\end{abstract}


The solvent relaxation response to the solute out of equilibrium plays a crucial role in determining rates and energetic of any physicochemical process in solution. ${ }^{1-6}$ In the past two decades, thanks to the rapid development of ultrafast spectroscopic techniques, an increasing interest has been devoted to the role played by the solvent on the ultrafast dynamics of photoexcited CT states, which are relevant in many photochemical and photophysical phenomena in different fields. ${ }^{7-11}$ Experimental research has been assisted by computational studies able to deliver atomistic insights into the solvent response dynamics and, in turn, on its impact on the electronic structure of the photo-excited solute. ${ }^{6,12-22}$ Within this framework, however, ultrafast experiments on solvated transition metal (TM) complexes are relatively few and recent, ${ }^{18,19,23-29}$ despite their ubiquitous employment in photovoltaics, optoelectronic devices and medical applications. ${ }^{30-35}$ Most of the published work focuses on polypyridine complexes, ${ }^{24,36}$ in particular on the prototype $\left[\mathrm{Ru}(\mathrm{bpy})_{3}\right]^{2+}$ complex, where the electron localization/delocalization dynamics following the photoinduced CT has been widely studied and debated. ${ }^{18,20,21,37-41}$ In the past years, solvent-dependent analysis of the transient absorption spectra, ${ }^{19,27,42-44}$ supported by computational modeling, ${ }^{20,21,45-47}$ suggested the idea of a rather complex solvent response dynamics, with the solvent as a principal actor in the modification of the solute's excited state charge distribution, often through the formation or breaking of strongly interacting solute-solvent clusters.

Recently, some of us reported joint experimental-computational studies on the excited state dynamics of the heteroleptic $(\mathrm{NCS})_{2} \mathrm{Ru}-\left(\mathrm{bpy}-(\mathrm{COOH})_{2}\right)\left(\mathrm{bpy}-\left(\mathrm{C}_{6} \mathrm{H}_{13}\right)_{2}\right), \mathrm{Z} 907$, dye, both in ethanol (EtOH) and dimethyl-sulfoxide (DMSO) solutions. ${ }^{27,48}$ Upon visible excitation at $520 \mathrm{~nm}$, the experimental transient absorption spectra (TAS) (see panels a) and b) in Figure 1) showed a positive signal around $650 \mathrm{~nm}$, attributed to the absorption of the triplet ligandmetal (NCS-Ru) to ligand (bpy-COOH) charge transfer $\left({ }^{3} \mathrm{LML}^{\prime} \mathrm{CT}\right)$ state, displayed in panel d), formed by ultrafast ( $<100 \mathrm{fs}$ ) intersystem crossing from the initially excited ${ }^{1} \mathrm{LML}^{\prime} \mathrm{CT}$ state. $^{49-51}$ While the signal in EtOH was progressively red-shifted and broadened in the [1 ps - $500 \mathrm{ps}$ ] range, no significant spectral changes were recorded in DMSO, suggesting that different solvent relaxation dynamics were responsible of the observed differences in the TAS evolutions. Even if theory might shed some light onto these phenomena, it is worth to stress that, 
from a computational point of view, a reliable modeling of TM complexes and their photochemical behavior is dramatically challenging. On the one hand, the feasibility of a pure quantummechanical (QM) approach ${ }^{27}$ is limited by both the system size (a relatively large number of solvent molecules should be accounted for) and the time scale (at least 500 ps) spanned by their dynamics. On the other hand, simulation techniques based on classical physics can seldom deliver a reasonable description of TM systems, in particular in their electronically excited states, due to the lack of standard force-fields (FFs) able to describe both the coordination around the metal center and the excited state vibrational dynamics. To tackle this problem, we developed ${ }^{48}$ two accurate quantum mechanical derived force fields (QMD-FFs), specifically parameterized for the ground $\left(\mathrm{S}_{0}\right)$ and lowest triplet $\left(\mathrm{T}_{1}\right)$ states of $\mathrm{Z} 907$ using state of the art techniques. ${ }^{41,52,53}$ Such QMD-FFs were validated by employing them in a multi-level protocol, to preliminary investigate the solvent effects on the steady-state absorption of the Z907 dye. ${ }^{48}$

Here we go farther, by addressing the modeling of the dynamic reorganization of the solvent out of equilibrium and its response to the sudden change in the solute's electronic distribution, to ultimately reproduce and rationalize the time evolution of the transient $\mathrm{T}_{1}$ absorption spectra of Z907 discussed above and displayed in Figure 1. To accomplish this challenging task, sufficiently long ( $\sim 1 \mathrm{~ns}$ ) non-equilibrium MD simulations based on the specific QMD-FFs ${ }^{48}$ have been carried out, followed by solvent response analysis and extensive transient electronic absorption spectra calculations. The pivotal idea is to mimic the excitation process from the ground $\mathrm{S}_{0}$ state to the first triplet state $\mathrm{T}_{1}$ with a sudden change of $\mathrm{FF}$, monitoring the subsequent system dynamics through nonequilibrium runs (hereafter referred as $\mathrm{T}_{1} @ \mathrm{EtOH}_{n e q}$ and $\left.\mathrm{T}_{1} @ \mathrm{DMSO}_{n e q}\right)$.

While the full protocol can be found in the Supporting Information, here we just resume the key points: i) 60 configurations are randomly extracted from the $\mathrm{S}_{0} @$ solvent $_{e q}$ NPT production runs; ${ }^{48}$ ii) the resulting configurations are employed as starting arrangements for further 60 NPT runs of $500 \mathrm{ps}$, using the $\mathrm{T}_{1}$ QMD-FF; iii) during each run, the relaxation of both solute and solvent nuclear coordinates, is monitored by storing the trajectories every 1 ps. The obtained frames were re-grouped into separate time intervals (ps), [0-1], [1-5], [5-20], [20-200] and [200-500]; iv) from $\approx 40$ to $\approx 100$ configurations are selected from each time interval, and 
used for the QM/MM excited state (unrestricted TD-B3LYP ${ }^{56}$ ) calculations of $\mathrm{T}_{1}$. The transient absorption spectra were then obtained by Gaussian convolution $(\sigma=0.1 \mathrm{eV})$ of the vertical excitation energies of the lowest $55 \mathrm{~T}_{1} \rightarrow \mathrm{T}_{n}$ transitions. In each configuration, the QM layer is composed of the solute and a certain number of solvent molecules, as detailed in the Supporting Information, while the MM layer is built by describing with point charges all the remaining solvent molecules within a radius of $15 \AA$ from the center of mass of Z907. To better elucidate the role of the solvent re-equilibration, $\mathrm{QM}$ calculations of the $\mathrm{T}_{1}$ vertical transition energies were carried out accounting for the solvent through three different schemes of increasing complexity: through a fully implicit continuum description (C-PCM), with an hybrid explicit/implicit scheme $\left(\mathrm{C}-\mathrm{PCM}_{h y b}\right)$ and a more complete scheme (QM/MM), where the solvent and its first solvation shell are accounted for at QM level, whereas the rest of solvent atoms are represented by point charges.

The thermal-averaged TAS in EtOH and DMSO, calculated with the QM/MM scheme by averaging the computed excitation energies over all the snapshots pertaining to a selected time interval, are plotted against the experimental counterpart and displayed in panels a) and b) of Figure 1. For the sake of clarity, all the experimental ${ }^{27}$ and calculated absorption maxima are gathered in Table 1. As is apparent in Figure 1 and reported in Ref. [27], the spectral evolutions in $\mathrm{EtOH}$ are quite different from those obtained in DMSO: while in the former an appreciable red-shift from $630-640 \mathrm{~nm}$ to $660-700 \mathrm{~nm}$ is observed along the monitored time delay, the recorded shift is negligible in the DMSO, presenting only small variations within the 635-648 $\mathrm{nm}$ range. Noticeably, the calculated absorption maxima (Table 1) in the QM/MM scheme almost quantitatively reproduce this picture, predicting only insignificant energy changes around 620-630 nm in DMSO and a clear shift at longer wavelengths (from 640 to $670 \mathrm{~nm}$ ) in EtOH. The remarkable agreement between the experimental (full lines) and calculated (dashed lines) TAS profiles is even more evident in Figure 1, where the blue (20-200) ps and green (200-500 ps) lines are, in both experiments and theory, sizably red-shifted and broadened for EtOH, while for DMSO the absorption maxima and band shapes remain essentially unchanged as the time delay increases.

By treating the medium at different level of approximation on the same solute's snapshots, 


\begin{tabular}{l|cccc|cc} 
& \multicolumn{5}{|c}{$\lambda_{\max }(\mathrm{nm})$} \\
\hline Time (ps) & \multicolumn{4}{|c}{ EtOH DMSO } \\
& $\exp ^{27}$ & C-PCM & C-PCM $_{\text {hyb }}$ & QM/MM & $\exp ^{27}$ & QM/MM \\
\hline \hline 1 & 631 & 641 & 636 & 632 & 634 & 622 \\
5 & 648 & 642 & 644 & 649 & 639 & 630 \\
20 & 666 & 634 & 638 & 640 & 646 & 625 \\
200 & 706 & 640 & 650 & 660 & 648 & 627 \\
500 & 682 & 640 & 648 & 671 & 640 & 623 \\
\hline \hline
\end{tabular}

Table 1: Thermal-averaged and experimental ${ }^{27}$ absorption maxima $(\mathrm{nm})$ of the ${ }^{3} \mathrm{MLL}^{\prime} \mathrm{CT}$ state in ETOH and DMSO. In the former solvent, three different solvation schemes are compared for the computed spectra: i) C-PCM; ii) C-PCM $\mathrm{Pyb}_{h b}$; iii) QM/MM.

we can access to more refined information about the origin of the aforementioned spectral shifts. First, as is apparent from panel c) in Figure 1), the calculations with an implicit solvent model (C-PCM) do not provide any shift in the triplet absorption maxima, calculated at about $640 \mathrm{~nm}$ (Table 1), allowing us to rule out the hypothesis that the predicted spectral evolutions might come from peculiar solute geometrical relaxations, induced by slow ethanol reorganization dynamics. Interestingly, when the first solvent shell surrounding the Z907 dye is explicitly included in the QM region, while the remaining solvent molecules are replaced by a continuum medium $\left(\mathrm{C}-\mathrm{PCM}_{h y b}\right)$, the effect is only partially captured, with the band maximum passing from about $640 \mathrm{~nm}$ at early times (1-20 ps) to about $650 \mathrm{~nm}$ after $20 \mathrm{ps}$. This finding is of pivotal importance here. Indeed, while the small spectral shifts occurring at short time delays (5 and 20 ps) are already well reproduced by including explicitly only the first solvation shell, to account for the larger shift and broadening characterizing the longer time delays (200 and $500 \mathrm{ps}$ ), it is necessary to also include the specific electrostatic embedding of the farther solvation shells. On these grounds one can thus infer that the origin of latest spectral shifts has to be found in slower reorganization dynamics of the second EtOH shell, which follows the faster solute-solvent hydrogen-bonds breaking involving the first shell upon the electronic excitation. Such an hypothesis seems to be supported by the results reported by Szymczak et al. in a non-equilibrium simulation of the photo-excitation of $\left.\left[\mathrm{Ru}(\mathrm{bpy})_{3}\right)\right]^{2+}$ in water, ${ }^{47}$ whereas the behavior observed in DMSO, confined to lighter reorganization effects within the first solvation 
shell, is more similar to the results obtained for $\left[\mathrm{Fe}(\mathrm{bmicp})_{2}\right]^{2+}$ in acetonitrile. ${ }^{54}$ With this information in mind, we will analyze the non-equilibrium solvation process by following the solvent dynamics around the complex at defined time intervals after the CT electronic excitation.

The atomic pair correlation functions $g_{\alpha \beta}(r)$ (see Ref.[48] and Supporting Information for definition) were computed for each time interval of the non-equilibrium MD trajectories and provide us with precise information on the solvent structure and its temporal evolution along the equilibration process. Considering that the local reorganization of solvent molecules around the solute in an aprotic medium is much less affected by the electronic transition, ${ }^{48}$ here we will limit the discussion to the EtOH case, while the same analysis in DMSO is reported in the Supporting Information. To monitor this reorganization around the excited solute, a reasonable choice is to follow the H-bonds network rearrangement, by calculating the $g_{S-H}(r)$ and $g_{O-H}(r)$ functions, and the ethanol structure around the metal center, by calculating the $g_{R u-H}(r)$ and $g_{R u-O}(r)$ functions. The top left panel of Figure 2 shows that the reorganization of the solutesolvent H-bond network around the NCS group following the electronic transition is ultrafast, as, over the first picosecond of simulation, the number of solvent hydrogen atoms close (and mostly H-bonded) to the Z907's Sulfur almost halves (black line) with respect to the population calculated ${ }^{48}$ for the ground state (dashed blue line). The partial CT to the carboxylic groups induces a concomitant strengthening of the COOH-EtOH H-bonds, evidenced by the sudden increase of the first neighbor peak in the $g_{\mathrm{OH}}(r)$ plot (bottom left panel of Figure 2). At 500 ps, the pair correlation function almost coincides with the one retrieved ${ }^{48}$ from the $\mathrm{T}_{1} @ \mathrm{EtOH}_{e q}$ run (green dashed lines), indicating that the solvent equilibration process has come to an end. In both functional groups, however, the net decrease/increase in the $\mathrm{SH} / \mathrm{OH}$ first shell population caused by the electronic excitation, is completed within the first three time intervals ( $20 \mathrm{ps}$ ), while only minor adjustments seem to take place thereafter. Nonetheless, we can expect that the breaking of the H-bonds between the solute NCS groups and the ethanol molecules within the first solvation shell (0-20 ps) will affect, at longer time delays (20-500 ps), the structure of the second solvation shell, as, indeed, shown by the increase of the second peak at $\sim 6 \AA$ in the top left panel of Figure 2. Similarly, slower reorganization effects, characterized by a decrease of the population of the first shell ( $\sim 4 \AA$ ) and an overall increase of the Ru-EtOH distance, also 
occur around the Ru atom (blue and green full lines in the top and bottom right panels of Figure 2), which undergoes a net charge depletion upon the electronic excitation.

The short- and long-range orientational ordering around the solute in the ground and excited state was farther investigated by computing, over the equilibrium trajectories $\mathrm{S}_{0} @$ solvent $_{e q}$ and $\mathrm{T}_{1} @$ solvent $_{e q}$, the $h^{110}$ orientational correlation function, defined in terms of the projection of the rotational invariant ${ }^{55}$ (see Supporting Information for a theoretical background). This function gives information about the orientation of the instantaneous molecular dipole of the solvent with respect to the solute's vector defined by either the C-S bond of the thiocyanate groups or the $\mathrm{C}-\mathrm{O}$ bond within the carboxyl group. Results are presented in Figure 3 for both solvents. In the protic solvent (left panel of Figure 3), the correlations around the C-O bond are less intense than those around the C-S bond, which is consistent with the results obtained for the pair correlation functions. The most striking effect is indeed the dramatic change in the orientational ordering around the thiocyanate groups. In the presence of a strong EtOHthiocyanate hydrogen bond in the ground state, we note a well defined double peak at distances consistent with those in the first shell. This double peak may be connected with quite a strong local alignment of solvent molecules, leading the ordering around one SCN group to be 'felt' by the second one. Moving to the excited state simulation, we observe that this strong orientational ordering definitely weakens (the corresponding function is much less structured). Regarding the correlations around the $\mathrm{C}-\mathrm{O}$ bond, a similar loss of ordering in the first shell is observed and a small negative peak become more intense at distances that are consistent with the (carboxyl) $\mathrm{O} \cdots \mathrm{HO}$ interaction. The corresponding analysis in aprotic solvent (right panel) shows that conversely the average orientation of DMSO molecules does not change upon the excitation in the solute and that the only effect is a slightly smaller population of solvent molecules in the first solvation shell.

The analysis discussed so far suggests the occurrence, at longer time delays, of a disruption of the local order in the second, and possibly farther solvation shells, induced by the ultrafast breaking of the NCS-EtOH H-bonds within the first solvation shell, in turn triggered by the markedly different solute's charge distribution. These findings can be corroborated by "visualizing" the solvent reorganization through the calculation of the spatial density functions (SDFs) 
of both the Hydrogen and Oxygen atoms of the ethanol $\mathrm{OH}$ group, for the $\mathrm{S}_{0}$ state and for each considered time interval following excitation. Within each interval, SDFs were averaged over 60 different runs, to improve statistics. Results are displayed in the top panel of Figure 4. Before excitation $\left(\mathrm{S}_{0}\right)$, the solvent is well structured around the NCS and COOH groups, with the network of H-bonds closely surrounding the $\mathrm{S}$ atom. Furthermore, even if more sparse (see also Figure $\mathrm{G}$ in the Supporting Information) a not negligible structured solvent density is also found around the metal, in agreement with the pair correlation functions displayed in Figure 2. Within the first $20 \mathrm{ps}$, this picture is completely perturbed as a consequence of the electronic transition and the related CT event: the charge depletion on the NCS ligands causes the progressive breaking of the H-bond network and the consequent decrease of the solvent density around the Sulfur atoms. On the same foot, the solvent mostly migrates to larger distances from the metal, starting to reside more often near the aromatic rings. As a consequence of these fast rearrangements, the solvent further relaxes, reaching a more structured reorganization only after 200 ps. In the 20-200 and 200-500 ps time intervals a re-orientation of the $\mathrm{OH}$ ethanol groups is clearly visible, with the Oxygen density (orange clouds) now oriented toward the Z907 and the Hydrogen atoms (violet density clouds) pointing instead toward the second neighbor shell, to possibly re-create H-bonds interactions. Indeed, this slow re-orientation is most likely capable to induce a rearrangement of the outer solvation spheres, which is revealed by the different long-range behavior of the $\mathrm{T}_{1}$ orientational functional, shown in Figure 3. Based on these findings, it should be expected that the solvent mean field felt by the solute and affecting its electronic behavior, changes along the monitored time intervals, even at longer time delays. This hypothesis is confirmed by computing from the point charge distribution (see Supporting Information for details) the average electrostatic potential experienced by the the Z907 dye at different time intervals, as shown in the bottom panels of Figure 4. The main effect (i.e. a decrease of about 0.03-0.04 a.u.) involves the NCS ligands, where the directionality of the solute-solvent interaction dictated by the H-bonds is lost and followed by a complete reorientation of the $\mathrm{EtOH}$ molecules, now pointing the Oxygens atoms toward the solute. A minor increase in the solvent mean field (about 0.01 a.u.) is also appreciable in on the BPY ligand bearing the $\mathrm{COOH}$ group, undergoing charge accumulation during the electronic excitation. These changes in the solvent mean field 
are compatibles with a blue-shift of the transient $\mathrm{T}_{1}$ absorption, arising from destabilization of the $\mathrm{T}_{13}$ excited state (see Figure $\mathrm{H}$ in the Supporting Information), which involves a CT from the reduced BPY-COOH to the Ru atom and a charge re-distribution on the NCS groups.

Concluding, by setting up a multilevel computational protocol, we have precisely simulated and fully rationalized the temporal evolution of experimental UV-Vis transient absorption spectra of the Z907 dye recorded in EtOH and DMSO. Noticeably, the spectral shifts occurring at long time delays (20-500 ps) in EtOH were shown to arise from slower reorganization dynamics of the second and farther solvation spheres following the ultrafast breaking of the network of solvent-solute H-bonds consequent to the electronic excitation to the ${ }^{1,3} \mathrm{LML}^{\prime} \mathrm{CT}$ state. The analysis of such solvent relaxation process along the excited state non-equilibrium dynamics unveiled two main "slow" phenomena modifying the average electrostatic potential acting on the solute: i) a global drift of the solvent away from the Ru center toward the second shell due to the mutated excited state charge distribution ; ii) a re-orientation of the solvent $\mathrm{OH}$ groups around the solute's NCS ligands after the breaking of the H-bonds, with the H atoms pointing toward the outer shell to possibly re-create the Hydrogen bonds.

Beside the remarkable agreement with the experimental spectra and the rationale proposed to interpret the specific Z907 TAS behavior at longer time delays, here we show that the consistent integration of different computational approaches in a sound multi-level procedure can be a powerful and reliable tool to achieve a deeper, atomistic, insight on the mechanisms underlying the electronic behavior of solvated TM complexes. This opens promising perspectives in the possibility of accurately treating the electrostatic and explicit solute-solvent interactions, which modify the solute's excited state density during the solvent relaxation process, finally delivering important information for the design of optimized photoactive molecular species.

\section{Acknowledgments}

MP thanks ANR JCJC HELIOSH2 (ANR-17-CE05-0007-01) for financial support and HPC resources from GENCI-CCRT/CINES (Grants 2018-A0010810139) and from the LPCT local computing clusters. 


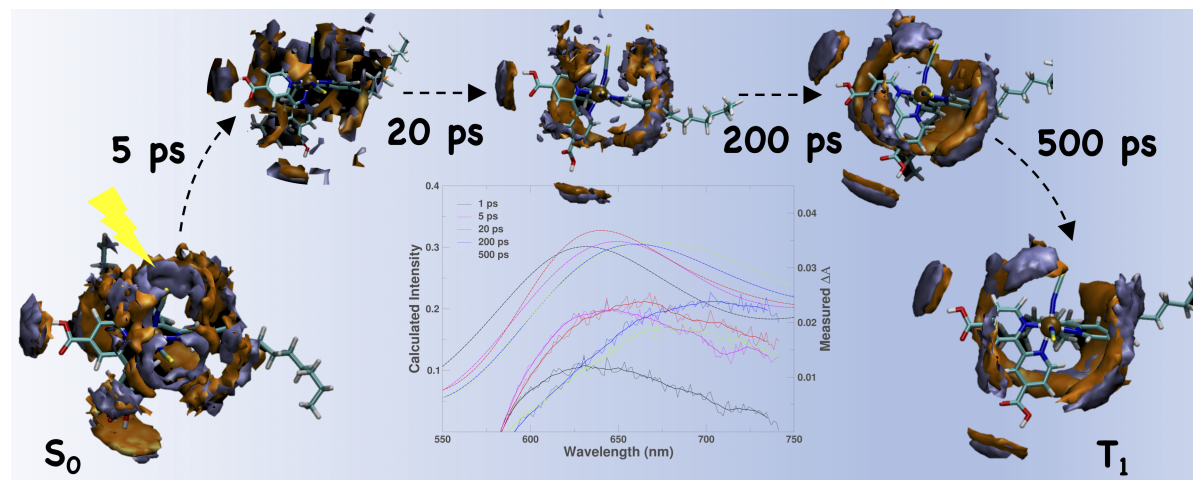

TOC graphic 
a)

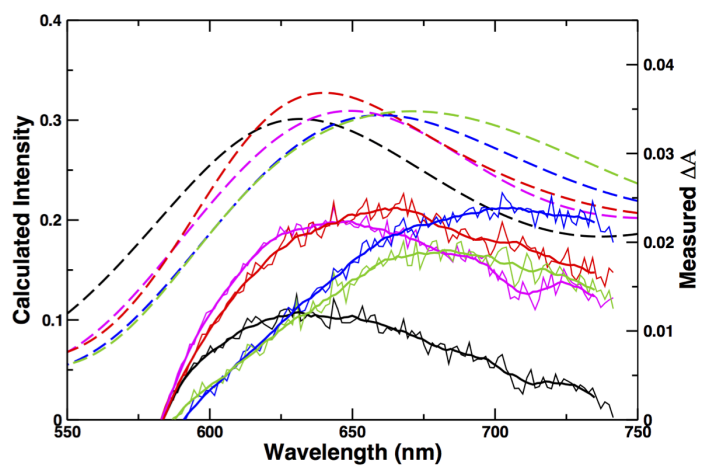

b)

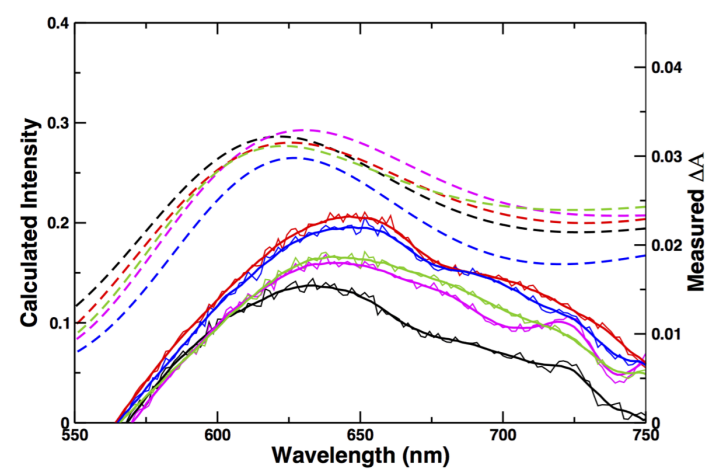

d)

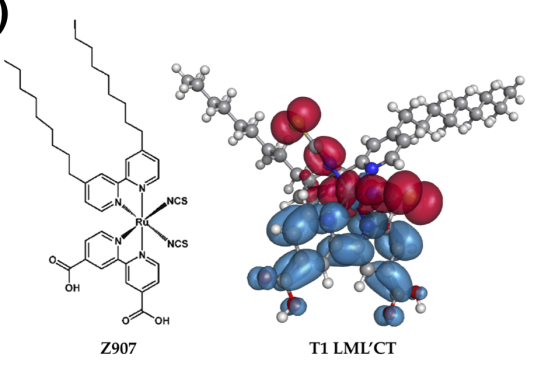

Figure 1: a) Calculated thermal-averaged (dashed lines) and experimental (full lines) transient absorption spectra in ETOH. The spectra are divided in time intervals, following the experimental time evolution, to monitor the effect of solvent reorganization as a function of time on the solute's optical response. The calculated spectra refers to the QM/MM solvation scheme. b) Calculated thermal-averaged (dashed lines) and experimental (full lines) transient absorption spectra in DMSO. c) Thermal-averaged transient absorption spectra in ETOH calculated using different solvation schemes: C-PCM (dashed lines), C-PCM ${ }_{h y b}$ (dotted-dashed lines and QM/MM (full lines). d) Chemical structure and iso-density plots (iso-value $5.0 \times 10^{-5}$ ) of CT characterizing the ${ }^{3} L M L^{\prime} C T$ of Z907. Red/blue lobes correspond to charge depletion/accumulation. 


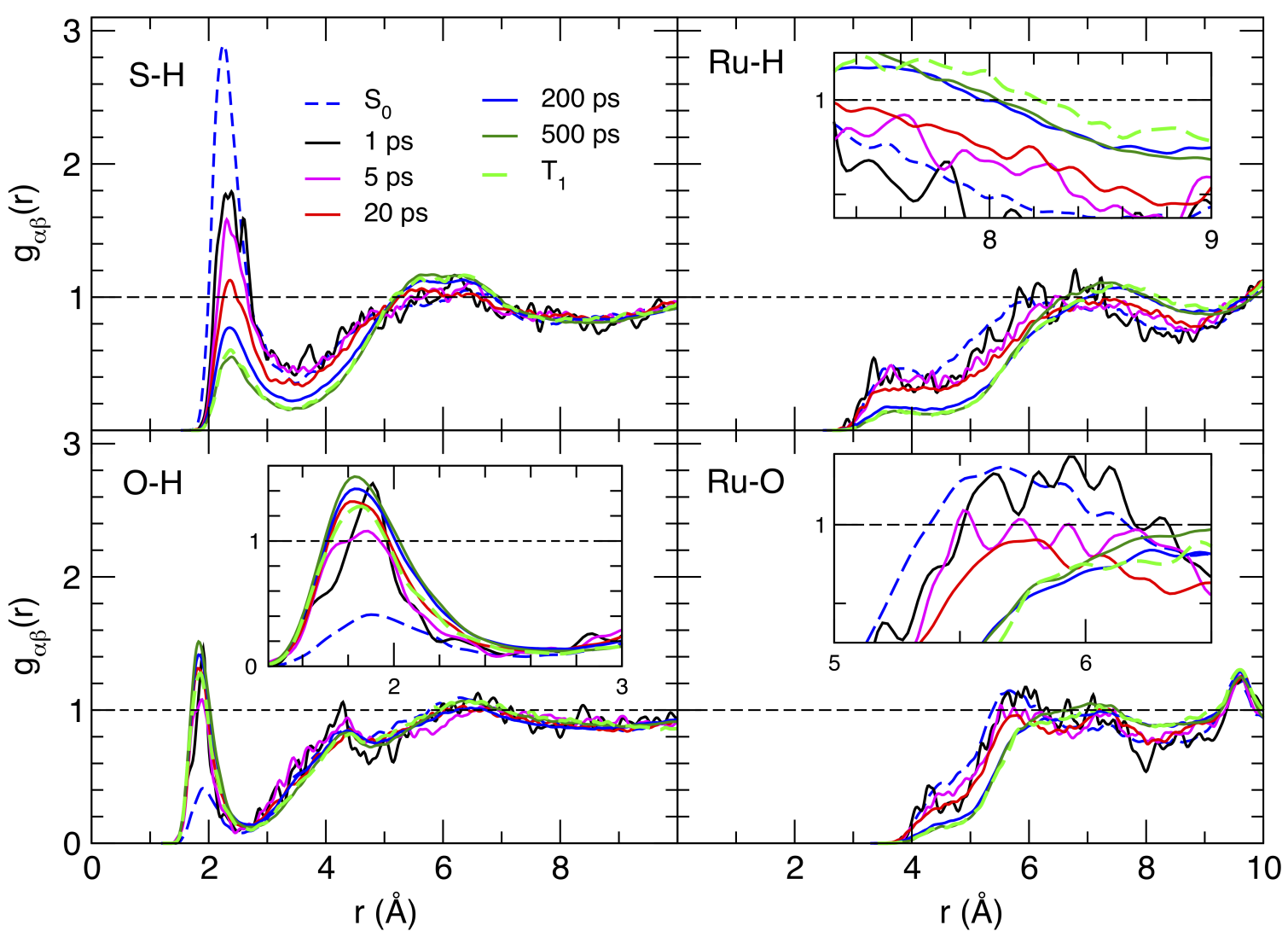

Figure 2: Atomic pair correlation functions $g_{\alpha \beta}(r)$ between an $\alpha$ atom of the solute (S $(\mathrm{O})$ atoms of the NCS $(\mathrm{COOH})$ groups in the left panels, $\mathrm{Ru}$ in the right ones) and a $\beta$ atom of the solvent hydroxy group ( $\mathrm{H}$ and $\mathrm{O}$ atoms in top and bottom panels, respectively). All functions displayed with full lines were computed for the time intervals over the $\mathrm{T}_{1} @ \mathrm{EtOH}_{n e q}$ trajectories. shown in the legend. The same functions computed for the $\mathrm{S}_{0} @ \mathrm{EtOH}_{e q}$ and $\mathrm{T}_{1} @ \mathrm{EtOH}_{e q}$ runs $^{48}$ are reported with blue and green dashed lines. 
a)

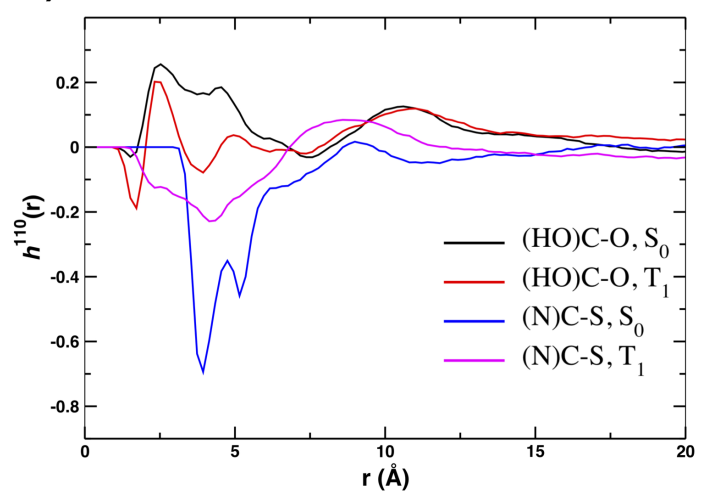

b)

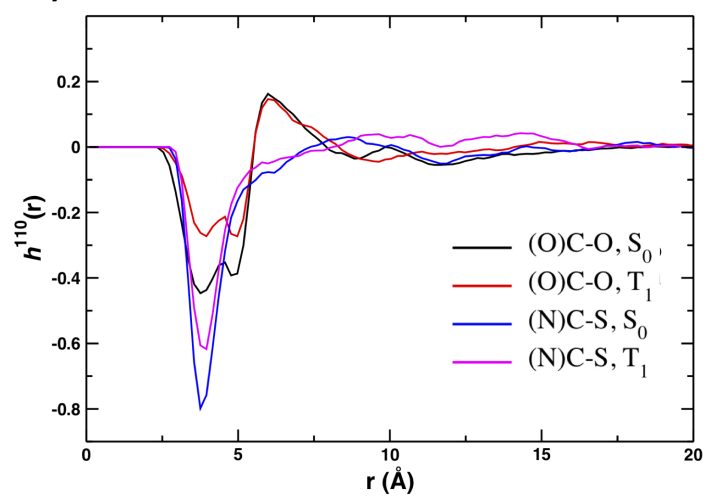

Figure 3: Orientational correlations for solvent molecules around the C-S and the C-O bonds of the solute in its ground and in its excited state (see text for more details).a) EtOH b) DMSO 

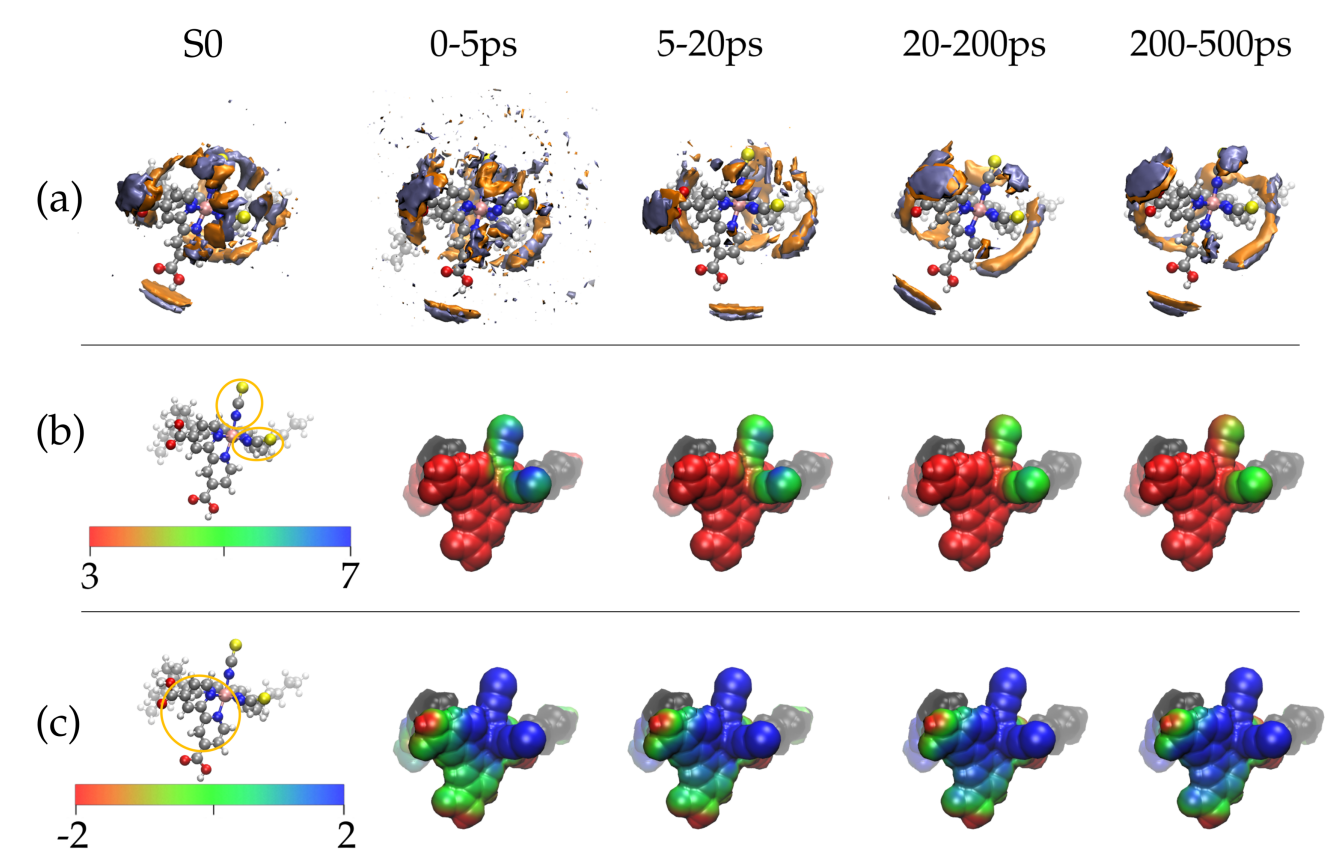

Figure 4: a: SDF's plots of the EtOH solvent molecules around the Z907 dye in different time intervals. Violet and orange density clouds are employed for solvent $\mathrm{H}$ and $\mathrm{O}$ atoms, respectively. $\mathrm{b}-\mathrm{c}$ : representation of the mean solvent $(\mathrm{EtOH})$ electrostatic potential $\left(10^{2}\right.$ a.u.) over the van der Waals surface the $\mathrm{Z} 907$ dye (excluding the alkyl chains). The color range is chosen so as to focus on the changes that arise on two different regions as indicated by yellow circles: the NCS groups (b) and the aromatic rings (c). 


\section{References}

[1] Kumpulainen, T.; Rosspeintner, A.; Vauthey, E. Probe dependence on polar solvation dynamics from fs broadband fluorescence Phys. Chem. Chem. Phys. 2017, 19, 8815-8825.

[2] Gould, E.-A.; Popov, A. V.; Tolbert, L. M.; Presiado, I.; Erez, Y.; Huppert, D.; Solntsev, K. M. Excited-state proton transfer in N-methyl-6-hydroxyquinolinium salts: solvent and temperature effects Phys. Chem. Chem. Phys. 2012, 14, 8964-8973.

[3] Pines, E.; Pines, D.; Ma, Y.-Z.; Fleming, G. R. Femtosecond Pump-Probe Measurements of Solvation by Hydrogen-Bonding Interactions ChemPhysChem 2004, 5, 1315-1327.

[4] Mohammed, O. F.; Dreyer, J.; Magnes, B.-Z.; Pines, E.; Nibbering, E. T. J. SolventDependent Photoacidity State of Pyranine Monitored by Transient Mid-Infrared Spectroscopy ChemPhysChem 2005, 6, 625-636.

[5] Mohammed, O.; Kwon, O.-H.; Othon, C.; Zewail, A. Charge Transfer Assisted by Collective Hydrogen-Bonding Dynamics Angew. Chem. Int. Ed. 2009, 48, 6251-6256.

[6] Barbara, P. F.; Meyer, T. J.; Ratner, M. A. Contemporary Issues in Electron Transfer Research J. Phys. Chem. 1996, 100, 13148-13168.

[7] Welin, E. R.; Le, C.; Arias-Rotondo, D. M.; McCusker, J. K.; MacMillan, D. W. C. Photosensitized, energy transfer-mediated organometallic catalysis through electronically excited nickel(II) Science 2017, 355, 380-385.

[8] Zhu, W.; Zheng, R.; Fu, X.; Fu, H.; Shi, Q.; Zhen, Y.; Dong, H.; Hu, W. Revealing the Charge-Transfer Interactions in Self-Assembled Organic Cocrystals: Two-Dimensional Photonic Applications Angew. Chem. Int. Ed. 54, 54, 6785-6789.

[9] Zhao, J.; Xu, K.; Yang, W.; Wang, Z.; Zhong, F. The triplet excited state of Bodipy: formation, modulation and application Chem. Soc. Rev. 2015, 44, 8904-8939.

[10] Fukuzumi, S.; Ohkubo, K.; Suenobu, T. Long-Lived Charge Separation and Applications in Artificial Photosynthesis Acc. Chem. Res. 2014, 47, 1455-1464. 
[11] Dong, H.; Wei, Y.; Zhang, W.; Wei, C.; Zhang, C.; Yao, J.; Zhao, Y. S. Broadband Tunable Microlasers Based on Controlled Intramolecular Charge-Transfer Process in Organic Supramolecular Microcrystals J. Am. Chem. Soc. 2016, 138, 1118-1121.

[12] Horng, M.; Gardecki, J.; Papazyan, A.; Maroncelli, M. Subpicosecond measurements of polar solvation dynamics: coumarin 153 revisited J. Phys. Chem. 1995, 99, 17311-17337.

[13] Maroncelli, M.; Fleming, G. R. Comparison of time-resolved fluorescence Stokes shift measurements to a molecular theory of solvation dynamics J. Chem. Phys. 1988, 89, 875881.

[14] Damrauer, N. H.; Cerullo, C. V.; Yeh, J. K.; Boussie; Shank; McCusker Femtosecond Dynamics of Excited-State Evolution in Science 1997, 275, 54-7.

[15] Heitele, H. Dynamic Solvent Effects on Electron-Transfer Reactions Angew. Chem. Int. Ed. 1993, 32, 359-377.

[16] Onfelt, B.; Lincoln, P.; Nordén, B.; Baskin, J. S.; Zewail, A. H. Femtosecond linear dichroism of DNA-intercalating chromophores: solvation and charge separation dynamics of [Ru(phen)2dppz]2+ systems. Proc. Natl. Acad. Sci. USA 2000, 97, 5708-13.

[17] Bhasikuttan, A. C.; Suzuki, M.; Nakashima, S.; Okada, T. Ultrafast Fluorescence Detection in Tris(2,2'-bipyridine)ruthenium(II) Complex in Solution: Relaxation Dynamics Involving Higher Excited States J. Am. Chem. Soc. 2002, 124, 8398-8405.

[18] Wallin, S.; Davidsson, J.; Modin, J.; Hammarström, L. Femtosecond Transient Absorption Anisotropy Study on $[\mathrm{Ru}(\mathrm{bpy}) 3] 2+$ and $[\mathrm{Ru}(\mathrm{bpy})(\mathrm{py}) 4] 2+$. Ultrafast Interligand Randomization of the MLCT State J. Phys. Chem. A 2005, 109, 4697-4704.

[19] Rondi, A.; Rodriguez, Y.; Feurer, T.; Cannizzo, A. Solvation-Driven Charge Transfer and Localization in Metal Complexes Acc. Chem. Res. 2015, 48, 1432-1440.

[20] Moret, M.-E.; Tavernelli, I.; Rothlisberger, U. Combined QM/MM and Classical Molecular Dynamics Study of $[\mathrm{Ru}($ bpy $)<\operatorname{sub}>3</$ sub $>]<\sup >2+</$ sup $>$ in Water J. Phys. Chem. B 2009, 113, 7737-7744. 
[21] Moret, M.-E.; Tavernelli, I.; Chergui, M.; Rothlisberger, U. Electron Localization Dynamics in the Triplet Excited State of $[\mathrm{Ru}(\mathrm{bpy})<\operatorname{sub}>3</$ sub $>]<\sup >2+</$ sup $>$ in Aqueous Solution Chem. Eur. J. 2010, 16, 5889-5894.

[22] van Driel, T. B.; Kjaer, K. S.; Hartsock, R. W.; Dohn, A. O.; Harlang, T.; Chollet, M.; Christensen, M.; Gawelda, W.; Henriksen, N. E.; Kim, J. G.; Haldrup, K.; Kim, K. H.; Ihee, H.; Kim, J.; Lemke, H.; Sun, Z.; Sundström, V.; Zhang, W.; Zhu, D.; Møller, K. B.; Nielsen, M. M.; Gaffney, K. J. Atomistic characterization of the active-site solvation dynamics of a model photocatalyst Nat. Commun. 2016, 7, 13678.

[23] El Nahhas, A.; Cannizzo, A.; van Mourik, F.; Blanco-Rodríguez, A. M.; Zális, S.; Vlček, A.; Chergui, M. Ultrafast Excited-State Dynamics of $[\operatorname{Re}(\mathrm{L})(\mathrm{CO}) 3(\mathrm{bpy})] \mathrm{n}$ Complexes: Involvement of the Solvent J. Phys. Chem. A 2010, 114, 6361-6369; PMID: 20465299.

[24] McCusker, J. K. Femtosecond Absorption Spectroscopy of Transition Metal ChargeTransfer Complexes Acc. Chem. Res. 2003, 36, 876-887; PMID: 14674779.

[25] Blanco-Rodríguez, A. M.; Ronayne, K. L.; Záliš, S.; Sýkora, J.; Hof, M.; Vlček, A. Solvation-Driven Excited-State Dynamics of [Re(4-Et-Pyridine)(CO)3(2,2'-bipyridine)]+ in Imidazolium Ionic Liquids. A Time-Resolved Infrared and Phosphorescence Study J. Phys. Chem. A 2008, 112, 3506-3514.

[26] Liu, L.; Duchanois, T.; Etienne, T.; Monari, A.; Beley, M.; Assfeld, X.; Haacke, S.; Gros, P. C. A new record excited state 3MLCT lifetime for metalorganic iron(ii) complexes Phys. Chem. Chem. Phys. 2016, 18, 12550-12556.

[27] Azzaroli, N.; Lobello, M. G.; Lapini, A.; Iagatti, A.; Bussotti, L.; Di Donato, M.; Calogero, G.; Pastore, M.; De Angelis, F.; Foggi, P. Monitoring the intramolecular charge transfer process in the Z907 solar cell sensitizer: a transient Vis and IR spectroscopy and ab initio investigation Phys. Chem. Chem. Phys. 2015, 17, 21594-21604.

[28] Leshchev, D.; Harlang, T. C. B.; Fredin, L. A.; Khakhulin, D.; Liu, Y.; Biasin, E.; Laursen, M. G.; Newby, G. E.; Haldrup, K.; Nielsen, M.; Wärnmark, K.; Sundström, V.; Persson, P.; 
Kjaer, K. S.; Wulff, M. Tracking the picosecond deactivation dynamics of a photoexcited iron carbene complex by time-resolved X-ray scattering Chem. Sci. 2018, 9, 405-414.

[29] Brown, A. M.; McCusker, C. E.; Carey, M. C.; Blanco-Rodríguez, A. M.; Towrie, M.; Clark, I. P.; Vlček, A.; McCusker, J. K. Vibrational Relaxation and Redistribution Dynamics in Ruthenium(II) Polypyridyl-Based Charge-Transfer Excited States: A Combined Ultrafast Electronic and Infrared Absorption Study J. Phys. Chem. A 2018, 122, 7941-7953.

[30] Listorti, A.; Durrant, J.; Barber, J. Solar to fuel Nature Mater. 2009, 8, 929-930.

[31] Sension, R. J. Quantum path to photosynthesis Nature 2007, 446, 740-741.

[32] Jailaubekov, A. E.; Willard, A. P.; Tritsch, J. R.; Chan, W.-L.; Sai, N.; Gearba, R.; Kaake, L. G.; Williams, K. J.; Leung, K.; Rossky, P. J.; Zhu, X.-Y. Hot charge-transfer excitons set the time limit for charge separation at donor/acceptor interfaces in organic photovoltaics Nature Mater. 2013, 12, 66-73.

[33] Grätzel, M. Conversion of sunlight to electric power by nanocrystalline dye-sensitized solar cells J. Photochem. Photobiol. A 2004, 164, 3-14.

[34] Zhang, M.; Murakami, T.; Ajima, K.; Tsuchida, K.; Sandanayaka, A. S. D.; Ito, O.; Iijima, S.; Yudasaka, M. Fabrication of $\mathrm{ZnPc/protein} \mathrm{nanohorns} \mathrm{for} \mathrm{double} \mathrm{photodynamic}$ and hyperthermic cancer phototherapy. PNAS 2008, 105, 14773-8.

[35] Cheng, L.; Wang, C.; Feng, L.; Yang, K.; Liu, Z. Functional Nanomaterials for Phototherapies of Cancer Chem. Rev. 2014, 114, 10869-10939.

[36] Juris, A.; Balzani, V.; Barigelletti, F.; Campagna, S.; Belser, P.; von Zelewsky, A. Ru(II) polypyridine complexes: photophysics, photochemistry, eletrochemistry, and chemiluminescence Coord. Chem. Rev. 1988, 84, 85-277.

[37] Damrauer, N. H.; McCusker, J. K. Ultrafast Dynamics in the Metal-to-Ligand Charge Transfer Excited-State Evolution of [Ru(4,4'-diphenyl-2,2'-bipyridine)3]2+ J. Phys. Chem. A 1999, 103, 8440-8446. 
[38] Yeh, A. T.; Shank, C. v.; McCusker, J. K. Ultrafast electron localization dynamics following photo-induced charge transfer Science 2000, 289, 935-938.

[39] Adam Webb, M.; Knorr, F. J.; McHale, J. L. Resonance Raman spectrum of $[\mathrm{Ru}$ (bipyridine) 3$] 2+$ in water, acetonitrile and their deuterated derivatives: the possible role of solvent in excited-state charge localization J. Raman Spectrosc. 2001, 32, 481485 .

[40] Benkö, G.; Kallioinen, J.; Myllyperkiö, P.; Trif, F.; Korppi-Tommola, J. E. I.; Yartsev, A. P.; Sundström, V. Interligand Electron Transfer Determines Triplet Excited State Electron Injection in RuN3 Sensitized TiO2 Films J. Phys. Chem. B 2004, 108, 2862-2867.

[41] Abedi, M.; Levi, G.; Zederkof, D. B.; Henriksen, N. E.; Pápai, M.; Møller, K. B. Excitedstate solvation structure of transition metal complexes from molecular dynamics simulations and assessment of partial atomic charge methods Phys. Chem. Chem. Phys. 2019, 21, 4082-4095.

[42] Indelli, M. T.; Bignozzi, C. A.; Scandola, F.; Collin, J.-P. Design of Long-Lived Ru(II) Terpyridine MLCT States. Tricyano Terpyridine Complexes Inorg. Chem. 1998, 37, 60846089; PMID: 11670747.

[43] Indelli, M. T.; Ghirotti, M.; Prodi, A.; Chiorboli, C.; Scandola, F.; McClenaghan, N. D.; Puntoriero, F.; Campagna, S. Solvent Switching of Intramolecular Energy Transfer in Bichromophoric Systems: Photophysics of (2,2Bipyridine)tetracyanoruthenate(II)/Pyrenyl Complexes Inorg. Chem. 2003, 42, 54895497; PMID: 12950195.

[44] Kjaer, K. S.; Kunnus, K.; Harlang, T. C. B.; Van Driel, T. B.; Ledbetter, K.; Hartsock, R. W.; Reinhard, M. E.; Koroidov, S.; Li, L.; Laursen, M. G.; Biasin, E.; Hansen, F. B.; Vester, P.; Christensen, M.; Haldrup, K.; Nielsen, M. M.; Chabera, P.; Liu, Y.; Tatsuno, H.; Timm, C.; Uhlig, J.; Sundstöm, V.; Németh, Z.; Szemes, D. S.; Bajnóczi, E.; Vankó, G.; Alonso-Mori, R.; Glownia, J. M.; Nelson, S.; Sikorski, M.; Sokaras, D.; Lemke, H. T.; 
Canton, S. E.; Wärnmark, K.; Persson, P.; Cordones, A. A.; Gaffney, K. J. Solvent control of charge transfer excited state relaxation pathways in [Fe(2,'-bipyridine $)(\mathrm{CN}) 4] 2-$ Phys. Chem. Chem. Phys. 2018, 20, 4238-4249.

[45] Lawson Daku, L. M.; Hauser, A. Ab Initio Molecular Dynamics Study of an Aqueous Solution of [Fe(bpy)3](Cl)2 in the Low-Spin and in the High-Spin States J. Phys. Chem. Lett. 2010, 1, 1830-1835.

[46] Hoff, D. A.; Silva, R.; Rego, L. G. C. Subpicosecond Dynamics of Metal-to-Ligand Charge-Transfer Excited States in Solvated $[\mathrm{Ru}(\mathrm{bpy}) 3] 2+$ Complexes The Journal of Physical Chemistry C 2011, 115, 15617-15626.

[47] Szymczak, J. J.; Hofmann, F. D.; Meuwly, M. Structure and dynamics of solvent shells around photoexcited metal complexes Phys. Chem. Chem. Phys. 2013, 15, 6268-6277.

[48] Prampolini, G.; Ingrosso, F.; Segalina, S., Alekos Caramori; Foggi, P.; Pastore, M. Dynamical and environmental effects on the optical properties of an heteroleptic $\mathrm{Ru}(\mathrm{II})$ polypyridine complex: a multilevel approach combining accurate ground and excited state QM-derived force fields, MD and TD-DFT J. Chem. Theory Comput. 2019, 15, 529-545.

[49] Touceda, P. T.; Vázquez, S. M.; Lima, M.; Lapini, A.; Foggi, P.; Dei, A.; Righini, R. Transient infrared spectroscopy: a new approach to investigate valence tautomerism $P$ hys. Chem. Chem. Phys. 2012, 14, 1038-1047.

[50] Yoon, S.; Kukura, P.; Stuart, C. M.; Mathies, R. A. Direct observation of the ultrafast intersystem crossing in tris(2,2'-bipyridine)ruthenium(II) using femtosecond stimulated Raman spectroscopy Mol. Phys. 2006, 104, 1275-1282.

[51] Ramakrishna, G.; Goodson, T.; Rogers-Haley, J. E.; Cooper, T. M.; McLean, D. G.; Urbas, A. Ultrafast Intersystem Crossing: Excited State Dynamics of Platinum Acetylide Complexes The Journal of Physical Chemistry C 2009, 113, 1060-1066.

[52] Cacelli, I.; Prampolini, G. Parametrization and Validation of Intramolecular Force Fields Derived from DFT Calculations J. Chem. Theory Comput. 2007, 3, 1803-1817. 
[53] Cerezo, J.; Prampolini, G.; Cacelli, I. Developing accurate intramolecular force fields for conjugated systems through explicit coupling terms Theor. Chem. Accounts 2018, 137, 80.

[54] Pápai, M.; Abedi, M.; Levi, G.; Biasin, E.; Nielsen, M. M.; Møller, K. B. Theoretical Evidence of Solvent-Mediated Excited-State Dynamics in a Functionalized Iron Sensitizer J. Phys. Chem. C 2019, 123, 2056-2065.

[55] Stell, G.; Patey, G.; Høye, J. Dielectric constants of fluid models: statistical mechanical theory and its quantitative implementation Adv. Chem. Phys., Volume 48 1981, 183-328.

[56] Becke, A. D. A new mixing of hartree-fock and local density-functional theories $J$. Chem. Phys. 1993, 98, 1372-1377. 